\title{
Multiplicity of Solutions for Schrödinger Equations with Concave-Convex Nonlinearities
}

\author{
Dong-Lun Wu, ${ }^{1,2}$ Chun-Lei Tang, ${ }^{1}$ and Xing-Ping Wu ${ }^{1}$ \\ ${ }^{1}$ School of Mathematics and Statistics, Southwest University, Chongqing 400715, China \\ ${ }^{2}$ College of Science, Southwest Petroleum University, Chengdu, Sichuan 610500, China
}

Correspondence should be addressed to Chun-Lei Tang; tangcl@swu.edu.cn

Received 10 July 2016; Accepted 1 November 2016

Academic Editor: Dong Ye

Copyright (C) 2016 Dong-Lun Wu et al. This is an open access article distributed under the Creative Commons Attribution License, which permits unrestricted use, distribution, and reproduction in any medium, provided the original work is properly cited.

We study the multiplicity of solutions for a class of semilinear Schrödinger equations: $-\Delta u+V(x) u=g(x, u)$, for $x \in \mathbb{R}^{N} ; u(x) \rightarrow$ 0 , as $|u| \rightarrow \infty$, where $V$ satisfies some kind of coercive condition and $g$ involves concave-convex nonlinearities with indefinite signs. Our theorems contain some new nonlinearities.

\section{Introduction and Main Results}

In this paper, we consider the multiplicity of solutions for the following semilinear Schrödinger equations:

$$
\begin{aligned}
-\Delta u+V(x) u & =g(x, u), \quad \text { for } x \in \mathbb{R}^{N}, \\
u(x) & \longrightarrow 0, \quad \text { as }|u| \longrightarrow \infty .
\end{aligned}
$$

Equation (1) has many applications in mathematical physics. For instance, in finding the standing wave solutions for the following nonlinear Schrödinger equation

$$
i \hbar \frac{\partial \varphi}{\partial t}=-\frac{\hbar^{2}}{2 m} \Delta \varphi+W(x) \varphi-b(x,|\varphi|) \varphi
$$

we can see that a standing wave solution of (2) is a solution of the form

$$
\varphi(x, t)=u(x) e^{-i E t / \hbar},
$$

where $i=\sqrt{-1}$. The function $\varphi(x, t)$ solves (2) if and only if $u(x)$ solves (1) with $V(x)=W(x)-E$ and $g(x, u)=b(x,|u|) u$.

The existence and multiplicity of solutions for problem (1) have been studied by many mathematicians in last two decades [1-36]. In 1992, Coti Zelati and Rabinowitz [7] obtained the existence of infinitely many solutions for problem (1) when $V(x)$ and $g(x, u)$ are both periodic in $x$ and $g(x, u)$ is supposed to satisfy the following so-called Ambrosetti-Rabinowitz superlinear condition.
(AR) there exists $\mu>2$ such that

$$
\begin{aligned}
& \operatorname{tg}(x, t) \geq \mu G(x, t)>0, \\
& \forall x \in \mathbb{R}^{N}, t \in \mathbb{R} \backslash\{0\}, \text { where } G(x, t)=\int_{0}^{t} g(x, v) d v .
\end{aligned}
$$

Condition (AR) provided a global growth condition of $g$ at both origin and infinity, which plays an important role in showing the boundedness of Palais-Smale sequences and the geometrical structure for the corresponding functional. But (AR) is so strict that many functions do not satisfy this condition. An usual and weaker superlinear condition is

(SQ) $G(x, u) /|u|^{2} \rightarrow \infty$ as $|u| \rightarrow \infty$ uniformly in $x \in \mathbb{R}^{N}$, which is first introduced by Liu and Wang [20] to obtain multiple solutions for superlinear elliptic equations and has been used by many mathematicians. Via a Nehari-type argument, Li et al. [19] obtained a ground state solution for problem (1) with the help of the following Nehari type assumption:

(Ne) $u \rightarrow g(x, u) /|u|$ is strictly increasing on $(-\infty, 0)$ and $(0,+\infty)$.

In a recent paper, $(\mathrm{Ne})$ is weakened by Liu [17] when the author treated a class of periodic Schrödinger equations. He made the following assumption:

$(\mathrm{WN}) u \rightarrow g(x, u) /|u|$ is increasing on $(-\infty, 0)$ and $(0,+\infty)$. 
After then, there are some papers [28, 35, 36] that obtained existence and multiplicity of nontrivial solutions for problem (1) with condition (WN). Recently, Tang [26] introduced a new superlinear condition.

(Ta) there exists a $\tau_{0} \in(0,1)$ such that

$$
\begin{array}{r}
\frac{1-\tau^{2}}{2} \operatorname{tg}(x, t) \geq \int_{\tau t}^{t} g(x, s) d s=G(x, t)-G(x, \tau t), \\
\forall \tau \in\left[0, \tau_{0}\right],(x, t) \in \mathbb{R}^{N} \times \mathbb{R} .
\end{array}
$$

With (Ta), Tang obtained the existence of ground state solutions for a class of superlinear Schrödinger equation involving some new nonlinearities. Motivated by the above works, in this paper, we shall study the multiplicity of solutions of problem (1) with concave-convex nonlinearities and the superlinear term satisfies some different growth assumptions from above. There are only few papers considering the concave-convex nonlinearities for problem (1). In [30], Wu considered problem (1) in a bounded domain with concave-convex nonlinearities and obtained two positive solutions when the weight function is indefinite in sign. After then, Wu [31] considered problem (1) in the entire space with sign-changing weight and obtained multiple positive solutions for problem (1). The results on multiple solutions for problem (1) with concave-convex nonlinearities can be also found in $[10,13]$. But in $[10,13,30,31]$, the authors only considered the specific nonlinearities. In this paper, we consider a more general case. The potential $V(x)$ satisfies the following coercive condition which is introduced by Bartsch and Wang in [4]:

$(V) V \in C\left(\mathbb{R}^{N}, \mathbb{R}\right), \inf _{x \in \mathbb{R}^{N}} V(x)>0$. There exists $\bar{r}>0$ such that

$$
\lim _{|y| \rightarrow \infty} \text { meas }\left\{x \in \mathbb{R}^{N}:|x-y| \leq \bar{r}, V(x) \leq M\right\}=0,
$$

$$
\forall M>0 \text {. }
$$

The main purpose of this paper is to obtain multiplicity of solutions for problem (1) with some new nonlinearities. The nonlinear term $g$ is considered to satisfy the following form:

$$
g(x, t)=\lambda f(x, t)+k(x, t) .
$$

Let $F(x, t)=\int_{0}^{t} f(x, v) d v$ and $K(x, t)=\int_{0}^{t} k(x, v) d v$. Now we state our main results.

Theorem 1. Suppose that (V), (7), and the following conditions hold:

$\left(g_{1}\right) F(x, t) \in C^{1}\left(\mathbb{R}^{N} \times \mathbb{R}, \mathbb{R}\right)$ and $F(x, 0)=0$.

$\left(g_{2}\right)$ There exit $\bar{x} \in \mathbb{R}^{N}, r_{0} \in(1,2)$, and $b_{0}>0$ such that $F(\bar{x}, t)>b_{0}|t|^{r_{0}}$ for all $t \in \mathbb{R}$.

$\left(g_{3}\right)$ For any $(x, t) \in \mathbb{R}^{N} \times \mathbb{R}$, there exist $r_{1}, r_{2} \in(1,2)$ such that

$$
|f(x, t)| \leq b_{1}(x)|t|^{r_{1}-1}+b_{2}(x)|t|^{r_{2}-1},
$$

where $b_{i}(x) \in L^{\beta_{i}}\left(\mathbb{R}^{N}, \mathbb{R}^{+}\right)$and $\beta_{i} \in\left(22^{*} /\left(2^{*}\left(2-r_{i}\right)+2^{*}-\right.\right.$ $\left.2), 2 /\left(2-r_{i}\right)\right]$ for $i=1,2$.

$\left(g_{4}\right) K(x, t)=a(x)|t|^{s}$, where $2<s<2^{*}$ and $a(x) \epsilon$ $L^{\infty}\left(\mathbb{R}^{N}, \mathbb{R}\right)$.

$\left(g_{5}\right)$ There exits $\Theta \subset \mathbb{R}$ such that $a(x)>0$ in $\Theta$ with meas $\Theta>0$.

Then, there exists $\lambda_{1}>0$ such that for any $\lambda \in\left(0, \lambda_{1}\right)$, problem (1) possesses at least two solutions.

Remark 2. Since $r_{i}>1$, we can see that $\beta_{i}>22^{*} /\left(2^{*}\left(2-r_{i}\right)+\right.$ $\left.2^{*}-2\right)>2^{*} /\left(2^{*}-r_{i}\right)$, which implies that $r_{i} \beta_{i}^{*}<2^{*}$, where $1 / \beta_{i}+1 / \beta_{i}^{*}=1$ for $i=1,2$.

Remark 3. It is easy to see that $\left(g_{4}\right)$ does not satisfy (AR), (SQ), (WN), and (Ta) since $a(x)$ can change sign.

Remark 4. In 2005, Liu and Wang [21] also considered problem (1) with concave-convex nonlinearities. But in their theorems, the nonlinear term was assumed to be a specific form, which is different from our theorem. Furthermore, it was required that $\int_{\mathbb{R}^{N}}(V(x))^{-1} d x<+\infty$ in [21], which is not needed in $(V)$.

Theorem 5. Suppose that $(V),(7),\left(g_{1}\right),\left(g_{3}\right)-\left(g_{5}\right)$, and the following condition hold:

$$
\left(g_{6}\right) F(x,-t)=F(x, t) \text { for all }(x, t) \in \mathbb{R}^{N} \times \mathbb{R} \text {. }
$$

Then, for any $\lambda \geq 0$, problem (1) possesses infinitely many solutions.

Remark 6. It is easy to see that $F(x, t)$ and $K(x, t)$ are both indefinite in signs. The sign-changing nonlinear terms have been studied by Tang [25]. But in [25], the author only considered the case $\lambda=0$ and $K(x, t)$ is positive when $|t|$ is large enough which is different from $\left(g_{5}\right)$.

Theorem 7. Suppose that $(V),(7),\left(g_{1}\right),\left(g_{2}\right),\left(g_{3}\right),\left(g_{5}\right)$, and the following conditions hold:

$\left(g_{7}\right) K \in C^{1}\left(\mathbb{R}^{N} \times \mathbb{R}, \mathbb{R}\right), K(x, 0)=0$ for all $x \in \mathbb{R}^{N}$.

$\left(g_{8}\right) K(x, t) / t^{2} \rightarrow+\infty$ as $|t| \rightarrow \infty$ uniformly in $x$.

$\left(g_{9}\right)$ There exist $\gamma>2$ and $d_{1}, \rho_{\infty}>0$ such that

$$
t k(x, t)-\gamma K(x, t) \geq-d_{1} t^{2}, \quad \forall x \in \mathbb{R}^{N},|t| \geq \rho_{\infty} .
$$

$\left(g_{10}\right)$ There exist $\zeta \in\left(2,2^{*}\right)$ and $d_{2}>0$ such that

$$
|k(x, t)| \leq d_{2}\left(|t|+|t|^{\zeta-1}\right), \quad \forall(x, t) \in \mathbb{R}^{N} \times \mathbb{R} .
$$

$\left(g_{11}\right) k(x, t)=o(|t|)$ as $t \rightarrow 0$ uniformly in $x$.

Then, there exists $\lambda_{2}>0$ such that for any $\lambda \in\left(0, \lambda_{2}\right)$, problem (1) possesses at least two solutions.

Remark 8 . There are functions satisfying the conditions of $\left(g_{7}\right)-\left(g_{11}\right)$, but not the condition $\left(g_{4}\right)$. For example, let

$$
K(x, t)=\left(1+\frac{t^{2}}{1+t^{2}}\right)|t|^{3} .
$$


Theorem 9. Suppose that $(V),(7),\left(g_{1}\right),\left(g_{3}\right),\left(g_{6}\right)-\left(g_{10}\right)$, and the following condition hold:

$\left(g_{12}\right)$ There exists $d_{3}>0$ such that $K(x, t) \geq-d_{3}|t|^{2}$ for all $x \in \mathbb{R}^{N}$.

$\left(g_{13}\right) K(x,-t)=K(x, t)$ for all $(x, t) \in \mathbb{R}^{N} \times \mathbb{R}$.

Then, for any $\lambda \geq 0$, problem (1) possesses infinitely many solutions.

Remark 10. In Theorem 9, we only need $\left(g_{9}\right)$ to hold when $|t|$ is large enough, which is different from the results in [25], in which the author required $\left(g_{9}\right)$ to hold in the entire space.

Remark 11. Obviously, it can be, respectively, deduced from (AR), (WN), and (Ta) that

(WSQ) $\operatorname{tg}(x, t)-2 G(x, t) \geq 0$ for all $(x, t) \in \mathbb{R}^{N} \times \mathbb{R}$.

However, (WSQ) cannot be deduced from the conditions of our theorems and there are functions to show this difference. For example, let $\lambda=0$ and

$$
\begin{aligned}
& K(x, t) \\
& \quad= \begin{cases}-|t|^{4}+|t|^{3}, & \text { for }|t| \leq \frac{4}{5}, \\
\left(|x|-\frac{4+4^{1 / 3}}{5}\right)^{4}+\frac{64-4^{4 / 3}}{625}, & \text { for }|t| \geq \frac{4}{5} .\end{cases}
\end{aligned}
$$

It is easy to see that (12) satisfies the conditions $\left(g_{7}\right)-\left(g_{12}\right)$, but not (WSQ).

In this paper, we will use the variational methods to prove our theorems. First, we introduce the definition of the (PS) ${ }^{*}$ condition and $(C)$ condition.

Definition 12. Let $E$ be a Hilbert space. A functional $I \in$ $C^{1}(E, \mathbb{R})$ is said to satisfy the (PS) ${ }^{*}$ condition with respect to $E_{j}, j=1,2, \ldots$, if any sequence $x_{j} \in E_{j}$ satisfying

$$
\begin{array}{r}
\left|I\left(x_{j}\right)\right|<\infty, \\
\left.I^{\prime}\right|_{E_{j}}\left(x_{j}\right) \longrightarrow 0
\end{array}
$$

implies a convergent subsequence, where $E_{j}$ is a sequence of linear subspace of $E$ with finite dimensional.

Definition 13. Let $E$ be a Hilbert space. A functional $I \in$ $C^{1}(E, \mathbb{R})$ is said to satisfy the $(C)$ condition if for any sequence $\left\{u_{n}\right\} \subset E$ satisfying $\left\{I\left(u_{n}\right)\right\}$ which is bounded and $\left\|I^{\prime}\left(u_{n}\right)\right\|(1+$ $\left.\left\|u_{n}\right\|\right) \rightarrow 0$ as $n \rightarrow \infty$ possesses a convergent subsequence.

In our proof, the Mountain Pass Theorem and the following critical points theorems are employed.

Lemma 14 (Lu [37]). Let $X$ be a real reflexive Banach space and $\Omega \subset X$ be a closed bounded convex subset of $X$. Suppose that $\varphi: X \rightarrow \mathbb{R}$ is a weakly lower semicontinuous (w.l.s.c. for short) functional. If there exists a point $x_{0} \in \Omega \backslash \partial \Omega$ such that

$$
\varphi(x)>\varphi\left(x_{0}\right), \quad \forall x \in \partial \Omega,
$$

then there must be a $x^{*} \in \Omega \backslash \partial \Omega$ such that

$$
\varphi\left(x^{*}\right)=\inf _{x \in \Omega} \varphi(x) .
$$

Lemma 15 (Chang [6]). Suppose that $I \in C^{1}(E, \mathbb{R})$ is even with $I(0)=0$ and that

$\left(C_{1}\right)$ there are constants $\varrho, \alpha>0$ and a finite dimensional linear subspace $X$ such that $\left.I\right|_{X^{\perp} \cap S_{e}} \geq \alpha$,

$\left(C_{2}\right)$ there is a sequence of linear subspace $\widetilde{X}_{m}, \operatorname{dim} \widetilde{X}_{m}=m$, and there exists $r_{m}>0$ such that

$$
I(u) \leq 0, \quad \text { on } \widetilde{X}_{m} \backslash B_{r(m)}, m=1,2, \ldots
$$

If, further, I satisfies the $(P S)^{*}$ condition with respect to $\left\{\widetilde{X}_{m} \mid m=1,2, \ldots\right\}$, then I possesses infinitely many distinct critical points corresponding to positive critical values.

\section{Preliminaries}

In this paper, we let

E

$$
:=\left\{u \in H^{1}\left(\mathbb{R}^{N}\right): \int_{\mathbb{R}^{N}}\left(|\nabla u|^{2}+V(x) u^{2}\right) d x<\infty\right\}
$$

with the inner product

$$
\langle u, v\rangle=\int_{\mathbb{R}^{N}}(\nabla u \cdot \nabla v+V(x) u v) d x
$$

and the norm $\|u\|=\langle u, u\rangle^{1 / 2}$. Then, $E$ is a Hilbert space. For any $1 \leq p<\infty$, we denote

$$
\begin{aligned}
\|u\|_{p} & =\left(\int_{\mathbb{R}^{N}}|u|^{p} d x\right)^{1 / p}, \\
\|u\|_{\infty} & =\operatorname{esssup}\left\{|u(x)|: x \in \mathbb{R}^{N}\right\} .
\end{aligned}
$$

The embedding theorem shows that $E \hookrightarrow L^{p}\left(\mathbb{R}^{N}\right)$ continuously for $p \in\left[2,2^{*}\right]$, which implies that there exists a constant $C_{p}>0$ such that

$$
\|u\|_{p} \leq C_{p}\|u\|
$$

for all $u \in E$. The corresponding functional is defined on $E$ as

$$
\begin{aligned}
I(u)= & \frac{1}{2} \int_{\mathbb{R}^{N}}\left(|\nabla u|^{2}+V(x) u^{2}\right) d x \\
& -\int_{\mathbb{R}^{N}} G(x, u) d x \\
= & \frac{1}{2}\|u\|^{2}-\lambda \int_{\mathbb{R}^{N}} F(x, u) d x-\int_{\mathbb{R}^{N}} K(x, u) d x .
\end{aligned}
$$

With condition $(V)$, we have the following compact embedding theorem. 
Lemma 16 (see [33]). Under assumption $(V)$, the embedding from $E$ into $L^{s}\left(\mathbb{R}^{N}\right)$ is compact for $2 \leq s<2^{*}$.

Lemma 17. Suppose that $(V),\left(g_{3}\right),\left(g_{7}\right)$, and $\left(g_{10}\right)$ hold; then, the functional $I$ is well defined and of $C^{1}$ class with

$$
\left\langle I^{\prime}(u), v\right\rangle=\langle u, v\rangle-\left\langle\psi^{\prime}(u), v\right\rangle-\left\langle\kappa^{\prime}(u), v\right\rangle,
$$

for all $v \in E$, where $\psi(u)=\int_{\mathbb{R}^{N}} F(x, u) d x$ and $\kappa(u)=$ $\int_{\mathbb{R}^{N}} K(x, u) d x$. Moreover, the critical points of $I$ in $E$ are solutions for problem (1).

Proof. By $\left(g_{3}\right),\left(g_{7}\right)$, and $\left(g_{10}\right)$, we have

$$
\begin{aligned}
& |F(x, t)| \leq \frac{1}{r_{1}} b_{1}(x)|t|^{r_{1}}+\frac{1}{r_{2}} b_{2}(x)|t|^{r_{2}}, \\
& |K(x, t)| \leq d_{2}\left(|t|^{2}+|t|^{\zeta}\right)
\end{aligned}
$$

for all $(x, t) \in \mathbb{R}^{N} \times \mathbb{R}$. It follows from $\left(g_{3}\right)$ and (20) that there exists $M_{1}>0$ such that

$$
\int_{\mathbb{R}^{N}}|F(x, u)| d x \leq M_{1}\left(\|u\|^{r_{1}}+\|u\|^{r_{2}}\right) .
$$

Then, we can deduce that

$$
\begin{aligned}
\int_{\mathbb{R}^{N}}|G(x, u)| d x \leq & \int_{\mathbb{R}^{N}}|\lambda F(x, u)+K(x, u)| d x \\
\leq & \lambda \int_{\mathbb{R}^{N}}|F(x, u)| d x \\
& +\int_{\mathbb{R}^{N}}|K(x, u)| d x \\
\leq & \lambda M_{1}\left(\|u\|^{r_{1}}+\|u\|^{r_{2}}\right) \\
& +d_{2} \int_{\mathbb{R}^{N}}\left(|u|^{2}+|u|^{\zeta}\right) d x \\
\leq & \lambda M_{1}\left(\|u\|^{r_{1}}+\|u\|^{r_{2}}\right) \\
& +d_{2}\left(C_{2}^{2}\|u\|^{2}+C_{\zeta}^{\zeta}\|u\|^{\zeta}\right)<\infty,
\end{aligned}
$$

which implies that $I$ is well defined. Similar to the proof of Proposition 2.2 in [34], we can see that $\psi \in C^{1}(E, \mathbb{R})$ and $\psi^{\prime}$ : $E \rightarrow E^{*}$ is compact. Obviously, $\kappa$ is also of $C^{1}$ class and $\kappa^{\prime}$ is compact, which means $I$ is of $C^{1}$ class and (22) holds. Finally, since $E$ is continuously embedded into $H^{1}\left(\mathbb{R}^{N}\right)$, a standard argument shows that all critical points of $I$ on $E$ are solutions of (1). We finish the proof of this lemma.

Remark 18. Lemma 17 still holds with $(V)$ and $\left(g_{4}\right)$ since the functions in $\left(g_{4}\right)$ satisfy $\left(g_{7}\right)$ and $\left(g_{10}\right)$.

By Lemma 17, we can easily obtain

$$
\begin{aligned}
\left\langle I^{\prime}(u), u\right\rangle= & \|u\|^{2}-\lambda \int_{\mathbb{R}^{N}} f(x, u) u d x \\
& -\int_{\mathbb{R}^{N}} k(x, u) u d x .
\end{aligned}
$$

\section{Proof of Theorem 1}

Subsequently, we show I possesses the conditions of the Mountain Pass Theorem.

Lemma 19. Suppose the conditions of Theorem 1 hold; then, there exist $\lambda_{1}, \varrho_{1}, \alpha_{1}>0$ such that $\left.I\right|_{\partial B_{\rho_{1}}} \geq \alpha_{1}$ for all $\lambda \in\left(0, \lambda_{1}\right)$, where $B_{Q_{1}}=\left\{u \in E:\|u\| \leq \varrho_{1}\right\}$.

Proof. It follows from (21), (25), $\left(g_{3}\right),\left(g_{4}\right)$, and (20) that

$$
\begin{aligned}
& I(u)=\frac{1}{2}\|u\|^{2}-\lambda \int_{\mathbb{R}^{N}} F(x, u) d x-\int_{\mathbb{R}^{N}} K(x, u) d x \\
& \quad \geq \frac{1}{2}\|u\|^{2}-\lambda M_{1}\left(\|u\|^{r_{1}}+\|u\|^{r_{2}}\right) \\
& \quad-\int_{\mathbb{R}^{N}} a(x)|u|^{s} d x \geq \frac{1}{2}\|u\|^{2}-\lambda M_{1}\left(\|u\|^{r_{1}}\right. \\
& \left.+\|u\|^{r_{2}}\right)-C_{s}^{s}\|a\|_{\infty}\|u\|^{s} \geq\left(\frac{1}{2}\right. \\
& \left.-\lambda M_{1}\left(\|u\|^{r_{1}-2}+\|u\|^{r_{2}-2}\right)-C_{s}^{s}\|a\|_{\infty}\|u\|^{s-2}\right)\|u\|^{2} .
\end{aligned}
$$

It is easy to see that there exist positive constants $\lambda_{1}, \varrho_{1}$, and $\alpha_{1}$ such that $\left.I\right|_{\partial B_{e_{1}}} \geq \alpha_{1}$ for all $\lambda \in\left(0, \lambda_{1}\right)$. We finish the proof of this lemma.

Lemma 20. Suppose the conditions of Theorem 1 hold; then, there exists $e_{1} \in E$ such that $\left\|e_{1}\right\|>\varrho$ and $I\left(e_{1}\right) \leq 0$, where $\varrho$ is defined in Lemma 19.

Proof. By Lusin's Theorem and $\left(g_{5}\right)$, there exists $\Sigma \subset \Theta$ such that $a(x)$ is continuous in $\Sigma$ with meas $\Sigma>(1 / 2)$ meas $\Theta>0$ with $\inf _{x \in \Sigma} a(x)>0$. We choose $\varphi_{1} \in C_{0}^{\infty}(\Sigma, \mathbb{R}) \backslash\{0\}$. Then, by (21), (25), $\left(g_{3}\right)$, and $\left(g_{4}\right)$, for any $\xi>0$, we obtain

$$
\begin{aligned}
I\left(\xi \varphi_{1}\right)= & \frac{\xi^{2}}{2} \int_{\Sigma}\left|\dot{\varphi}_{1}\right|^{2} d x-\lambda \int_{\Sigma} F\left(x, \xi \varphi_{1}\right) d x \\
& -\xi^{s} \int_{\Sigma} a(x)\left|\varphi_{1}\right|^{s} d x \\
\leq & \frac{\xi^{2}}{2} \int_{\Sigma}\left|\dot{\varphi}_{1}\right|^{2} d x \\
& +\lambda M_{1}\left(\xi^{r_{1}}\left\|\varphi_{1}\right\|^{r_{1}}+\xi^{r_{2}}\left\|\varphi_{1}\right\|^{r_{2}}\right) \\
& -\xi^{s} a_{0} \int_{\Sigma}\left|\varphi_{1}\right|^{s} d x
\end{aligned}
$$

where $a_{0}=\inf _{x \in \Sigma} a(x)$, which implies that

$$
I_{1}\left(\xi \varphi_{1}\right) \longrightarrow-\infty, \quad \text { as } \xi \longrightarrow+\infty \text {. }
$$

Therefore, there exists $\xi_{1}>0$ such that $I_{1}\left(\xi_{1} \varphi_{1}\right)<0$. Let $e_{1}=$ $\xi_{1} \varphi_{1}$, we can see $I\left(e_{1}\right)<0$, which proves this lemma. 
Lemma 21. Suppose the conditions of Theorem 1 hold; then, I satisfies the $(C)$ condition.

Proof. Assume that $\left\{u_{n}\right\}_{n \in \mathbb{N}} \subset E$ is a sequence such that $\left\{I\left(u_{n}\right)\right\}$ is bounded and $\left\|I^{\prime}\left(u_{n}\right)\right\|\left(1+\left\|u_{n}\right\|\right) \rightarrow 0$ as $n \rightarrow \infty$. Then, there exists a constant $M_{2}>0$ such that

$$
\begin{aligned}
\left|I\left(u_{n}\right)\right| & \leq M_{2}, \\
\left\|I^{\prime}\left(u_{n}\right)\right\|\left(1+\left\|u_{n}\right\|\right) & \leq M_{2} .
\end{aligned}
$$

Subsequently, we show that $\left\{u_{n}\right\}$ is bounded in $E$. Arguing in an indirect way, we assume that $\left\|u_{n}\right\| \rightarrow \infty$ as $n \rightarrow \infty$. It follows from (31), (27), (21), (23), $\left(g_{3}\right)$, and $\left(g_{4}\right)$ that there exist $M_{3}, M_{4}>0$ such that

$$
\begin{aligned}
o(1)= & \frac{(s+1) M_{2}}{\left\|u_{n}\right\|^{2}} \geq \frac{s I\left(u_{n}\right)+\left\|I^{\prime}\left(u_{n}\right)\right\|\left(1+\left\|u_{n}\right\|\right)}{\left\|u_{n}\right\|^{2}} \\
\geq & \frac{s I\left(u_{n}\right)-\left\langle I^{\prime}\left(u_{n}\right), u_{n}\right\rangle}{\left\|u_{n}\right\|^{2}} \\
= & \left(\frac{s}{2}-1\right)-\frac{\lambda \int_{\mathbb{R}^{N}} s F\left(x, u_{n}\right)-f\left(x, u_{n}\right) u_{n} d x}{\left\|u_{n}\right\|^{2}} \\
\geq & \left(\frac{s}{2}-1\right) \\
& -\frac{\lambda M_{3} \int_{\mathbb{R}^{N}} b_{1}(x)\left|u_{n}\right|^{r_{1}}+b_{2}(x)\left|u_{n}\right|^{r_{2}} d x}{\left\|u_{n}\right\|^{2}} \\
\geq & \left(\frac{s}{2}-1\right)-\lambda M_{4}\left(\left\|u_{n}\right\|^{r_{1}-2}+\left\|u_{n}\right\|^{r_{2}-2}\right) \\
\longrightarrow & \left(\frac{s}{2}-1\right), \quad \text { as } n \longrightarrow \infty,
\end{aligned}
$$

which is a contradiction. Hence, $\left\{u_{n}\right\}$ is bounded in $E$. Then, there exists a subsequence, still denoted by $\left\{u_{n}\right\}$, such that $u_{n} \rightarrow u$ in $E$. Therefore,

$$
\left\langle I^{\prime}\left(u_{n}\right)-I^{\prime}(u), u_{n}-u\right\rangle \longrightarrow 0, \quad \text { as } n \longrightarrow+\infty .
$$

Let $\sigma_{i}=2 /\left(r_{i}-1\right)$ and $\eta_{i}>0$ satisfying $1 / \beta_{i}+1 / \sigma_{i}+1 / \eta_{i}=1$, where $i=1,2$. By $\left(g_{3}\right)$, we can see that $\eta_{i} \in\left[2,2^{*}\right)$ for $i=1,2$. It follows from (20) and Lemma 16 that

$$
\begin{aligned}
& \int_{\mathbb{R}^{N}}\left(f\left(x, u_{n}\right)-f(x, u), u_{n}-u\right) d x \leq \int_{\mathbb{R}^{N}} \mid f\left(x, u_{n}\right) \\
& \quad-f(x, u)|| u_{n}-u \mid d x \\
& \quad \leq \int_{\mathbb{R}^{N}}\left(b_{1}(x)\left(\left|u_{n}\right|^{r_{1}-1}+|u|^{r_{1}-1}\right)\right. \\
& \left.\quad+b_{2}(x)\left(\left|u_{n}\right|^{r_{2}-1}+|u|^{r_{2}-1}\right)\right)\left|u_{n}-u\right| d x \\
& \quad \leq\left\|b_{1}\right\|_{\beta_{1}}\left(\left\|u_{n}\right\|_{2}^{r_{1}-1}+\|u\|_{2}^{r_{1}-1}\right)\left\|u_{n}-u\right\|_{\eta_{1}}+\left\|b_{2}\right\|_{\beta_{2}} \\
& \quad \cdot\left(\left\|u_{n}\right\|_{2}^{r_{2}-1}+\|u\|_{2}^{r_{2}-1}\right)\left\|u_{n}-u\right\|_{\eta_{2}} \longrightarrow 0,
\end{aligned}
$$$$
\text { as } n \longrightarrow \infty \text {. }
$$

Similarly, we have

$$
\begin{aligned}
& \int_{\mathbb{R}^{N}}\left(k\left(x, u_{n}\right)-k(x, u), u_{n}-u\right) d x \\
& \quad \leq \int_{\mathbb{R}^{N}}\left|k\left(x, u_{n}\right)-k(x, u)\right|\left|u_{n}-u\right| d x \\
& \quad=s \int_{\mathbb{R}^{N}}|a(x)|\left(\left|u_{n}\right|^{s-1}+|u|^{s-1}\right)\left|u_{n}-u\right| d x \\
& \quad \leq s\|a\|_{\infty}\left(\left\|u_{n}\right\|_{s}^{s-1}+\|u\|_{s}^{s-1}\right)\left\|u_{n}-u\right\|_{s} \longrightarrow 0, \\
& \text { as } n \longrightarrow \infty .
\end{aligned}
$$

It follows from (27) that

$$
\begin{aligned}
& \left\langle I^{\prime}\left(u_{n}\right)-I^{\prime}(u), u_{n}-u\right\rangle \\
& =\left\|u_{n}-u\right\|^{2} \\
& \quad-\lambda \int_{\mathbb{R}^{N}}\left(f\left(x, u_{n}\right)-f(x, u), u_{n}-u\right) d x \\
& \quad-\int_{\mathbb{R}^{N}}\left(k\left(x, u_{n}\right)-k(x, u), u_{n}-u\right) d x,
\end{aligned}
$$

which implies that $\left\|u_{n}-u\right\| \rightarrow 0$ as $n \rightarrow+\infty$. Then, $I$ satisfies the $(C)$ condition.

Lemma 22. Suppose that the conditions of Theorem 1 hold; then, there exists a critical point of I corresponding to negative critical value.

Proof. By Lemma 19, we can see that there exists a local minimizer of $I$ in $B_{Q_{1}}$, the following proof is to show this minimizer is not zero. By $\left(g_{1}\right)$ and $\left(g_{2}\right)$, there exists $\sigma_{3}>0$ such that

$$
F(\bar{x}, t)>\frac{1}{2} b_{0}|t|^{r_{0}}
$$

for all $x \in \Upsilon_{\sigma_{3}}(\bar{x})$ and $t \in \mathbb{R}$, where $\Upsilon_{\sigma_{3}}(\bar{x})=\left\{x \in \mathbb{R}^{N}\right.$ : $\left.|x-\bar{x}| \leq \sigma_{3}\right\}$. Choosing $\varphi_{2} \in C_{0}^{\infty}\left(\Upsilon_{\sigma_{3}}(\bar{x}), \mathbb{R}\right) \backslash\{0\}$, it follows from (21), (37), and $\left(g_{4}\right)$ that

$$
\begin{aligned}
I\left(\theta \varphi_{2}\right)= & \frac{\theta^{2}}{2}\left\|\varphi_{2}\right\|^{2}-\lambda \int_{\mathbb{R}^{N}} F\left(x, \theta \varphi_{2}\right) d x \\
& -\theta^{s} \int_{\mathbb{R}^{N}} a(x)\left|\varphi_{2}\right|^{s} d x \\
\leq & \frac{\theta^{2}}{2}\left\|\varphi_{2}\right\|^{2}-\frac{\theta^{r_{0}}}{2} \lambda b_{0} \int_{\Upsilon_{\sigma_{3}}(\bar{x})}\left|\varphi_{2}\right|^{r_{0}} d x \\
& +\theta^{s}\|a\|_{\infty} \int_{\Upsilon_{\sigma_{3}(\bar{x})}\left|\varphi_{2}\right|^{s} d x<0}
\end{aligned}
$$

for $\theta>0$ small enough. By Lemmas 19 and 14, there exists $U_{0} \in B_{Q_{1}} \backslash \partial B_{Q_{1}}$ such that

$$
\begin{aligned}
I\left(U_{0}\right) & =\inf _{u \in B_{\rho_{1}}} I(u)<0<\alpha_{1}, \\
I^{\prime}\left(U_{0}\right) & =0 .
\end{aligned}
$$

The proof of this lemma is finished. 
From Lemmas 19-22, we can see that problem (1) possesses at least two solutions.

\section{Proof of Theorem 5}

Lemma 23. Suppose the conditions of Theorem 5 hold; then, I satisfies $\left(C_{1}\right)$.

Proof. Let $\left\{v_{j}\right\}_{j=1}^{\infty}$ be a completely orthogonal basis of $E$ and $X_{k}=\bigoplus_{j=1}^{k} S_{j}$, where $S_{j}=\operatorname{span}\left\{v_{j}\right\}$. For any $q \in\left[2,2^{*}\right)$, we set

$$
h_{k}(q)=\sup _{u \in X_{k}^{\perp},\|u\|=1}\|u\|_{q} .
$$

It follows from Lemma 2.10 in [25] that $h_{k}(q) \rightarrow 0$ as $k \rightarrow \infty$ for any $q \in\left[2,2^{*}\right)$. Set

$$
\begin{aligned}
H_{k}= & \frac{\lambda}{r_{1}} h_{k}^{r_{1}}\left(r_{1} \beta_{1}^{*}\right)\left\|b_{1}\right\|_{\beta_{1}}+\frac{\lambda}{r_{2}} h_{k}^{r_{2}}\left(r_{2} \beta_{2}^{*}\right)\left\|b_{2}\right\|_{\beta_{2}} \\
& +h_{k}^{s}(s)\|a\|_{\infty} .
\end{aligned}
$$

Then, there exists $k_{0}>0$ such that $H_{k} \leq 1 / 4$ for all $k \geq k_{0}$. Then, for any $u \in X_{k_{0}}^{\perp} \cap \partial B_{\varrho}$ with $0<\varrho \leq 1$, it follows from (21), $\left(g_{3}\right),(23),\left(g_{4}\right)$, and $(40)$ that

$$
\begin{aligned}
I(u)= & \frac{1}{2}\|u\|^{2}-\lambda \int_{\mathbb{R}^{N}} F(x, u) d x-\int_{\mathbb{R}^{N}} a(x)|u|^{s} d x \\
\geq & \frac{1}{2}\|u\|^{2}-\frac{\lambda}{r_{1}} \int_{\mathbb{R}^{N}} b_{1}(x)|u|^{r_{1}} d x \\
& -\frac{\lambda}{r_{2}} \int_{\mathbb{R}^{N}} b_{2}(x)|u|^{r_{2}} d x-\|a\|_{\infty} \int_{\mathbb{R}^{N}}|u|^{s} d x \\
\geq & \frac{1}{2}\|u\|^{2}-\frac{\lambda}{r_{1}} h_{k_{0}}^{r_{1}}\left(r_{1} \beta_{1}^{*}\right)\left\|b_{1}\right\|_{\beta_{1}}\|u\|^{r_{1}} \\
& -\frac{\lambda}{r_{2}} h_{k_{0}}^{r_{2}}\left(r_{2} \beta_{2}^{*}\right)\left\|b_{2}\right\|_{\beta_{2}}\|u\|^{r_{2}} \\
& -h_{k_{0}}^{s}(s)\|a\|_{\infty}\|u\|^{s} \geq \frac{1}{2}\|u\|^{2}-H_{k_{0}}\|u\| \\
\geq & \frac{1}{2}\|u\|^{2}-\frac{1}{4}\|u\| .
\end{aligned}
$$

Hence, (42) shows that there exist $\alpha_{2}>0$ and $\varrho_{2} \in(0,1)$ such that $\left.I\right|_{X_{k_{0}}^{\perp} \cap \partial B_{e_{2}}} \geq \alpha_{2}$. We finish the proof of this lemma.

Lemma 24. Suppose the conditions of Theorem 5 hold; then, I satisfies $\left(C_{2}\right)$.

Proof. Let $\Sigma$ and $a_{0}$ be as defined in Lemma 20. Then, it is easy to see that $W_{0}^{1,2}(\Sigma, \mathbb{R}) \subset E$ and $W_{0}^{1,2}(\Sigma, \mathbb{R})$ is a Hilbert space. We can choose a sequence completely orthogonal basis $\left\{e_{j}\right\}_{j=1}^{\infty} \subset W_{0}^{1,2}(\Sigma, \mathbb{R})$. Let $R_{j}=\operatorname{span}\left\{e_{j}\right\}$ and $\widetilde{X}_{m}=\bigoplus_{j=1}^{m} R_{j}$. Then, for any $u_{m} \in \widetilde{X}_{m}$, we have supp $u_{m} \subset \Sigma$, where $\operatorname{supp} u_{m}=\overline{\left\{x \in \mathbb{R}^{N}: u_{m}(x) \neq 0\right\}}$. Since $\operatorname{dim} \widetilde{X}_{m}=m$, there exists a constant $T_{m}>0$ such that

$$
\|u\|_{s} \geq T_{m}\|u\|
$$

for all $u \in \widetilde{X}_{m}$. We can deduce from (21), (25), $\left(g_{4}\right)$, and (43) that

$$
\begin{aligned}
I\left(u_{m}\right)= & \frac{1}{2}\left\|u_{m}\right\|^{2}-\lambda \int_{\mathbb{R}^{N}} F\left(x, u_{m}\right) d x \\
& -\int_{\mathbb{R}^{N}} K\left(x, u_{m}\right) d x \\
\leq & \frac{1}{2}\left\|u_{m}\right\|^{2}+\lambda M_{1}\left(\left\|u_{m}\right\|^{r_{1}}+\left\|u_{m}\right\|^{r_{2}}\right) \\
& -\int_{\Sigma} a(x)\left|u_{m}\right|^{s} d x \\
\leq & \frac{1}{2}\left\|u_{m}\right\|^{2}+\lambda M_{1}\left(\left\|u_{m}\right\|^{r_{1}}+\left\|u_{m}\right\|^{r_{2}}\right) \\
& -T_{m}^{s} a_{0}\left\|u_{m}\right\|^{s} .
\end{aligned}
$$

Then, there exists $r(m)>0$ such that $I\left(u_{m}\right) \leq 0$ for all $u_{m} \in$ $\widetilde{X}_{m} \backslash B_{r(m)}$, which proves this lemma.

The proof of the following lemma is similar to Lemma 21; we omit it here.

Lemma 25. Suppose the conditions of Theorem 5 hold; then, I satisfies the $(P S)^{*}$ condition.

Then, by Lemma 15, we can deduce that $I$ possesses infinitely many critical points, which implies that problem (1) has infinitely many solutions.

\section{Proof of Theorem 7}

Lemma 26. Suppose the conditions of Theorem 7 hold; then, there exist $\lambda_{2}, \varrho_{3}, \alpha_{3}>0$ such that $\left.I\right|_{\partial B_{\varrho_{3}}} \geq \alpha_{3}$ for all $\lambda \in\left(0, \lambda_{2}\right)$.

Proof. By $\left(g_{7}\right),\left(g_{10}\right)$, and $\left(g_{11}\right)$, for any $\varepsilon>0$, there exists $D_{\varepsilon}>0$ such that

$$
|K(x, t)| \leq \varepsilon|t|^{2}+D_{\varepsilon}|t|^{\zeta}, \quad \forall(x, t) \in \mathbb{R}^{N} \times \mathbb{R} .
$$

It follows from (21), (45), (25), $\left(g_{3}\right)$, and (20) that

$$
\begin{aligned}
& I(u)=\frac{1}{2}\|u\|^{2}-\lambda \int_{\mathbb{R}^{N}} F(x, u) d x-\int_{\mathbb{R}^{N}} K(x, u) d x \\
& \geq \frac{1}{2}\|u\|^{2}-\lambda M_{1}\left(\|u\|^{r_{1}}+\|u\|^{r_{2}}\right)-\varepsilon \int_{\mathbb{R}^{N}}|u|^{2} d x \\
& \quad-D_{\varepsilon} \int_{\mathbb{R}^{N}}|u|^{\zeta} d x \geq \frac{1}{2}\|u\|^{2}-\lambda M_{1}\left(\|u\|^{r_{1}}\right. \\
& \left.+\|u\|^{r_{2}}\right)-\varepsilon C_{2}^{2}\|u\|^{2}-D_{\varepsilon} C_{\zeta}^{\zeta}\|u\|^{\zeta}=\left(\left(\frac{1}{2}-\varepsilon C_{2}^{2}\right)\right. \\
& \left.-\lambda M_{1}\left(\|u\|^{r_{1}-2}+\|u\|^{r_{2}-2}\right)-D_{\varepsilon} C_{\zeta}^{\zeta}\|u\|^{\zeta-2}\right)\|u\|^{2} .
\end{aligned}
$$

Letting $\varepsilon<1 / 2 C_{2}^{2}$, there exist positive constants $\lambda_{2}, \varrho_{3}$, and $\alpha_{3}$ such that $\left.I\right|_{\partial B_{e_{3}}} \geq \alpha_{3}$ for all $\lambda \in\left(0, \lambda_{2}\right)$. 
Lemma 27. Suppose the conditions of Theorem 7 hold; then, there exists $e_{2} \in E$ such that $\left\|e_{2}\right\|>\varrho_{3}$ and $I\left(e_{2}\right) \leq 0$, where $\varrho_{3}$ is defined in Lemma 26.

Proof. Set $e_{3} \in C_{0}^{\infty}\left(\Upsilon_{1}(0), \mathbb{R}\right)$ such that $\left\|e_{3}\right\|=1$, where $\Upsilon$ is defined in Lemma 22. For $M_{5}>\left(2 \int_{Y_{1}(0)}\left|e_{3}\right|^{2} d x\right)^{-1}$, it follows from $\left(g_{8}\right)$ that there exist $Q>0$ such that

$$
K(x, t) \geq M_{5} t^{2}
$$

for all $x \in \Upsilon_{1}(0)$ and $|t|>Q$. It follows from $\left(g_{7}\right)$ and $\left(g_{11}\right)$ that there exists $\rho_{1}>0$ such that

$$
|K(x, t)| \leq|t|^{2}
$$

for all $|t| \leq \rho_{1}$ and $x \in \mathbb{R}^{N}$. It follows from $\left(g_{8}\right)$ and (48) that there exists $d_{4}>0$ such that

$$
K(x, t) \geq-d_{4} t^{2}
$$

for all $(x, t) \in \mathbb{R}^{N} \times \mathbb{R}$. Then, we can deduce from (47) and (49) that

$$
K(x, t) \geq M_{5}\left(t^{2}-Q^{2}\right)-d_{4} Q^{2}
$$

for all $(x, t) \in \Upsilon_{1}(0) \times \mathbb{R}$. By (21), (50), (20), and (25), for every $\eta \in \mathbb{R}^{+}$, we have

$$
\begin{aligned}
I\left(\eta e_{3}\right)= & \frac{\eta^{2}}{2}\left\|e_{3}\right\|^{2}-\lambda \int_{\mathbb{R}^{N}} F\left(x, \eta e_{3}\right) d x \\
& -\int_{\mathbb{R}^{N}} K\left(x, \eta e_{3}\right) d x \\
\leq & \frac{\eta^{2}}{2}+\lambda M_{1}\left(\eta^{r_{1}}\left\|e_{3}\right\|^{r_{1}}+\eta^{r_{2}}\left\|e_{3}\right\|^{r_{2}}\right) \\
& -\int_{\Upsilon_{1}(0)} M_{5}\left(\left|\eta e_{3}\right|^{2}-Q^{2}\right)-d_{4} Q^{2} d x \\
\leq & \left(\frac{1}{2}-M_{5} \int_{\Upsilon_{1}(0)}\left|e_{3}\right|^{2} d x\right) \eta^{2} \\
& +\lambda M_{1}\left(\eta^{r_{1}}\left\|e_{3}\right\|^{r_{1}}+\eta^{r_{2}}\left\|e_{3}\right\|^{r_{2}}\right) \\
& +\left(M_{5}+d_{4}\right) Q^{2} \text { meas } \Upsilon_{1}(0),
\end{aligned}
$$

which implies that

$$
I\left(\eta e_{3}\right) \longrightarrow-\infty, \quad \text { as } \eta \longrightarrow+\infty .
$$

Therefore, there exists $\eta_{1}>0$ such that $I\left(\eta_{1} e_{3}\right)<0$ and $\left\|\eta_{1} e_{3}\right\|>\varrho_{3}$. Let $e_{2}=\eta_{1} e_{3}$, we can see $I\left(e_{2}\right)<0$, which proves this lemma.

Lemma 28. Suppose the conditions of Theorem 7 hold; then, I satisfies the $(P S)$ condition.

Proof. Assume that $\left\{u_{n}\right\}_{n \in \mathbb{N}} \subset E$ is a sequence such that

$$
\begin{gathered}
\left|I\left(u_{n}\right)\right|<\infty, \\
I^{\prime}\left(u_{n}\right) \longrightarrow 0,
\end{gathered}
$$

Then, there exists a constant $M_{6}>0$ such that

$$
\begin{aligned}
\left|I\left(u_{n}\right)\right| & \leq M_{6}, \\
\left\|I^{\prime}\left(u_{n}\right)\right\|_{E^{*}} & \leq M_{6} .
\end{aligned}
$$

Subsequently, we show that $\left\{u_{n}\right\}$ is bounded in $E$. Set

$$
\widetilde{K}(x, t)=t k(x, t)-\gamma K(x, t),
$$

where $\gamma$ is defined in $\left(g_{9}\right)$. Arguing in an indirect way, we assume that $\left\|u_{n}\right\| \rightarrow+\infty$ as $n \rightarrow \infty$. Set $z_{n}=u_{n} /\left\|u_{n}\right\|$; then, $\left\|z_{n}\right\|=1$, which implies that there exists a subsequence of $\left\{z_{n}\right\}$, still denoted by $\left\{z_{n}\right\}$, such that $z_{n} \rightarrow z_{0}$ in $E$ and $z_{n} \rightarrow z_{0}$ uniformly on $\mathbb{R}^{N}$ as $n \rightarrow \infty$. The following discussion is divided into two cases.

Case $1\left(z_{0} \neq \equiv 0\right)$. Let $\Omega=\left\{x \in \mathbb{R}^{N}|| z_{0}(x) \mid>0\right\}$. Then, we can see that meas $(\Omega)>0$. Since $\left\|u_{n}\right\| \rightarrow+\infty$ as $m \rightarrow \infty$ and $\left|u_{n}\right|=\left|z_{n}\right| \cdot\left\|u_{n}\right\|$; then, we have $\left|u_{n}\right| \rightarrow+\infty$ as $n \rightarrow \infty$ for a.e. $x \in \Omega$. On one hand, it follows from (21), (25), and (54) that

$$
\begin{aligned}
& \left|\int_{\mathbb{R}^{N}} \frac{K\left(x, u_{n}\right)}{\left\|u_{n}\right\|^{2}} d x-\frac{1}{2}\right| \\
& \quad=\left|\frac{I\left(u_{n}\right)}{\left\|u_{n}\right\|^{2}}+\lambda \int_{\mathbb{R}^{N}} \frac{F\left(x, u_{n}\right)}{\left\|u_{n}\right\|^{2}} d x\right| \\
& \leq \frac{M_{6}}{\left\|u_{n}\right\|^{2}}+\frac{\lambda M_{1}\left(\left\|u_{n}\right\|^{r_{1}}+\left\|u_{n}\right\|^{r_{2}}\right)}{\left\|u_{n}\right\|^{2}} \longrightarrow 0, \\
& \quad \text { as } n \longrightarrow \infty,
\end{aligned}
$$

which implies that

$$
\lim _{n \rightarrow \infty} \int_{\mathbb{R}^{N}} \frac{K\left(x, u_{n}\right)}{\left\|u_{n}\right\|^{2}} d x=\frac{1}{2} .
$$

On the other hand, by $\left(g_{8}\right),(49)$, and Fatou's Lemma, we can obtain

$$
\begin{aligned}
& \lim _{n \rightarrow \infty} \int_{\mathbb{R}^{N}} \frac{K\left(x, u_{n}\right)}{\left\|u_{n}\right\|^{2}} d x \\
& \quad \geq \lim _{n \rightarrow \infty} \int_{\Omega} \frac{K\left(x, u_{n}\right)}{\left\|u_{n}\right\|^{2}} d x-d_{4} \lim _{n \rightarrow \infty} \int_{\mathbb{R}^{N} \backslash \Omega}\left|z_{n}\right|^{2} d x \\
& \quad \geq \lim _{n \rightarrow \infty} \int_{\Omega} \frac{K\left(x, u_{n}\right)}{\left|u_{n}\right|^{2}}\left|z_{n}\right|^{2} d x-d_{4} C_{2}^{2}=+\infty,
\end{aligned}
$$

which contradicts (57).

Case $2\left(z_{0} \equiv 0\right)$. By $\left(g_{10}\right)$, we can deduce that

$$
|t k(x, t)| \leq d_{2}\left(|t|^{2}+|t|^{\zeta}\right)
$$

for all $(x, t) \in \mathbb{R}^{N} \times \mathbb{R}$, which implies that

$$
\begin{aligned}
|\widetilde{K}(x, t)| & =|t k(x, t)-\gamma K(x, t)| \\
& \leq d_{2}(1+\gamma)\left(|t|^{2}+|t|^{\zeta}\right)
\end{aligned}
$$


for all $(x, t) \in \mathbb{R}^{N} \times \mathbb{R}$. It follows from (54), (21), (25), $\left(g_{9}\right)$, $\left(g_{3}\right),(20),(60)$, and Sobolev's embedding theorem that

$$
\begin{aligned}
& o(1)=\frac{\gamma M_{6}+M_{6}\left\|u_{n}\right\|}{\left\|u_{n}\right\|^{2}} \geq \frac{\gamma I\left(u_{n}\right)-\left\langle I^{\prime}\left(u_{n}\right), u_{n}\right\rangle}{\left\|u_{n}\right\|^{2}} \\
& \geq\left(\frac{\gamma}{2}-1\right) \\
& -\frac{\lambda\left(\gamma \max \left\{1 / r_{1}, 1 / r_{2}\right\}+1\right)}{\left\|u_{n}\right\|^{2}}\left(C_{r_{1} \beta_{1}^{*}}^{r_{1}}\left\|b_{1}\right\|_{\beta_{1}}\left\|u_{n}\right\|^{r_{1}}\right. \\
& \left.+C_{r_{2} \beta_{2}^{*}}^{r_{2}}\left\|b_{2}\right\|_{\beta_{2}}\left\|u_{n}\right\|^{r_{2}}\right)+\frac{1}{\left\|u_{n}\right\|^{2}} \int_{\mathbb{R}^{N}} \widetilde{K}\left(x, u_{n}\right) d x \\
& =\left(\frac{\gamma}{2}-1\right)+\frac{1}{\left\|u_{n}\right\|^{2}}\left(\int_{\left\{x \in \mathbb{R}^{N} \| u_{n} \mid \leq \rho_{\infty}\right\}} \widetilde{K}\left(x, u_{n}\right) d x\right. \\
& \left.+\int_{\left\{x \in \mathbb{R}^{N}|| u_{n} \mid>\rho_{\infty}\right\}} \widetilde{K}\left(x, u_{n}\right) d x\right)+o(1) \geq\left(\frac{\gamma}{2}-1\right) \\
& -\frac{1}{\left\|u_{n}\right\|^{2}}\left(d_{2}(1+\gamma)\right. \\
& \cdot \int_{\left\{x \in \mathbb{R}^{N}|| u_{n} \mid \leq \rho_{\infty}\right\}}\left(\left|u_{n}\right|^{2}+\left|u_{n}\right|^{\zeta}\right) d x \\
& \left.+\int_{\left\{x \in \mathbb{R}^{N}|| u_{n} \mid>\rho_{\infty}\right\}} d_{1}\left|u_{n}\right|^{2} d x\right)+o(1) \geq\left(\frac{\gamma}{2}-1\right) \\
& -\frac{1}{\left\|u_{n}\right\|^{2}}\left(d_{2}(1+\gamma)\left(1+\rho_{\infty}^{\zeta-2}\right)\right. \\
& \cdot \int_{\left\{x \in \mathbb{R}^{N}|| u_{n} \mid \leq \rho_{\infty}\right\}}\left|u_{n}\right|^{2} d x \\
& \left.+\int_{\left\{x \in \mathbb{R}^{N}|| u_{n} \mid>\rho_{\infty}\right\}} d_{1}\left|u_{n}\right|^{2} d x\right)+o(1) \geq\left(\frac{\gamma}{2}-1\right) \\
& -\left(d_{2}(1+\gamma)\left(1+\rho_{\infty}^{\zeta-2}\right)+d_{1}\right) \int_{\mathbb{R}^{N}}\left|z_{n}\right|^{2} d x+o(1) \\
& \longrightarrow\left(\frac{\gamma}{2}-1\right), \quad \text { as } n \longrightarrow \infty,
\end{aligned}
$$

which is a contradiction. Hence, $\left\{u_{n}\right\}$ is bounded in $E$. The following proof is similar to Lemma 21 . Then, $I$ satisfies the (C) condition.

It follows from the Mountain Pass Theorem that there exists a critical point $\widehat{u}_{0}$ such that $I\left(\widehat{u}_{0}\right) \geq \alpha_{3}$ and $I^{\prime}\left(\widehat{u}_{0}\right)=0$, where $\alpha_{3}$ is defined in Lemma 26. Subsequently, we look for the second critical point of $I$ by Lemma 14 .

Lemma 29. Suppose that the conditions of Theorem 7 hold; then, there exists a critical point of I corresponding to negative critical value.

Proof. Since we have (45), the proof of this lemma is similar to Lemma 22.
Then, problem (1) possesses at least two solutions. The proof of Theorem 7 is finished.

\section{Proof of Theorem 9}

In this section, we use Lemma 15 to prove Theorem 9.

Lemma 30. Suppose the conditions of Theorem 9 hold; then, I satisfies $\left(C_{1}\right)$.

Proof. Let $X_{k}$ and $h_{k}(q)$ be as defined in Lemma 23. For any $u \in X_{k}^{\perp} \cap \partial B_{\rho}$ with $0<\varrho \leq 1$, it follows from (21), (23), (45), (20), and (40) that

$$
\begin{gathered}
I(u)=\frac{1}{2}\|u\|^{2}-\lambda \int_{\mathbb{R}^{N}} F(x, u) d x-\int_{\mathbb{R}^{N}} K(x, u) d x \\
\geq \frac{1}{2}\|u\|^{2}-\frac{\lambda}{r_{1}} \int_{\mathbb{R}^{N}} b_{1}(x)|u|^{r_{1}} d x-\frac{\lambda}{r_{2}} \\
\cdot \int_{\mathbb{R}^{N}} b_{2}(x)|u|^{r_{2}} d x-d_{2} \int_{\mathbb{R}^{N}}\left(|u|^{2}+|u|^{\zeta}\right) d x \\
\geq \frac{1}{2}\|u\|^{2}-\frac{\lambda}{r_{1}} h_{k}^{r_{1}}\left(r_{1} \beta_{1}^{*}\right)\left\|b_{1}\right\|_{\beta_{1}}\|u\|^{r_{1}}-\frac{\lambda}{r_{2}} \\
\cdot h_{k}^{r_{2}}\left(r_{2} \beta_{2}^{*}\right)\left\|b_{2}\right\|_{\beta_{2}}\|u\|^{r_{2}}-d_{2}\left(h_{k}^{2}(2)\|u\|^{2}\right. \\
\left.+h_{k}^{\zeta}(\zeta)\|u\|^{\zeta}\right) \geq \frac{1}{2}\|u\|^{2}-\left(\frac{\lambda}{r_{1}} h_{k}^{r_{1}}\left(r_{1} \beta_{1}^{*}\right)\left\|b_{1}\right\|_{\beta_{1}}\right. \\
\left.+\frac{\lambda}{r_{2}} h_{k}^{r_{2}}\left(r_{2} \beta_{2}^{*}\right)\left\|b_{2}\right\|_{\beta_{2}}+d_{2} h_{k}^{2}(2)+d_{2} h_{k}^{\zeta}(\zeta)\right)\|u\| .
\end{gathered}
$$

The following proof is similar to Lemma 23 . Hence, $I$ satisfies $\left(C_{1}\right)$. We finish the proof of this lemma.

Lemma 31. Suppose the conditions of Theorem 9 hold; then, I satisfies $\left(C_{2}\right)$.

Proof. Set $\widetilde{X}_{m}=\bigoplus_{j=1}^{m} S_{j}$, where $S_{j}$ is defined in Lemma 23 . For any $u \in \widetilde{X}_{m} \backslash\{0\}$ and $\vartheta>0$, set

$$
\Gamma_{\vartheta}(u)=\left\{x \in \mathbb{R}^{N}:|u| \geq \mathcal{V}\|u\|\right\} .
$$

Similar to Lemma 2.4 in [34], there exists $\vartheta_{0}>0$ such that

$$
\operatorname{meas}\left(\Gamma_{\vartheta_{0}}(u)\right) \geq \vartheta_{0}
$$

for all $u \in \widetilde{X}_{m}$. By $\left(g_{8}\right)$, there exist $\xi>0$ such that

$$
\begin{aligned}
K(x, u) & \geq \frac{1 / 2+d_{3} C_{2}^{2}+1}{\vartheta_{0}^{3}}|u|^{2} \\
& \geq \frac{1 / 2+d_{3} C_{2}^{2}+1}{\vartheta_{0}}\|u\|^{2}
\end{aligned}
$$


for all $u \in \widetilde{X}_{m}$ and $x \in \Gamma_{\vartheta_{0}}(u)$ with $\|u\| \geq \xi$, where $d_{3}$ is defined in $\left(g_{12}\right)$. Choosing $\varsigma_{m}>\xi$, then for any $u \in \widetilde{X}_{m} \backslash B_{\varsigma_{m}}$, it follows from (21), (20), $\left(g_{12}\right),(25),(64),\left(g_{3}\right)$, and (65) that

$$
\begin{aligned}
I(u)= & \frac{1}{2}\|u\|^{2}-\lambda \int_{\mathbb{R}^{N}} F(x, u) d x-\int_{\mathbb{R}^{N}} K(x, u) d x \\
\leq & \frac{1}{2}\|u\|^{2}-\lambda \int_{\mathbb{R}^{N}} F(x, u) d x \\
& -\int_{\Gamma_{\vartheta_{0}}(u)} K(x, u) d x+d_{3} \int_{\mathbb{R}^{N} \backslash \Gamma_{\vartheta_{0}}(u)}|u|^{2} d x \\
\leq & \frac{1}{2}\|u\|^{2}+\lambda M_{1}\left(\|u\|^{r_{1}}+\|u\|^{r_{2}}\right) \\
& -\frac{1 / 2+d_{3} C_{2}^{2}+1}{\vartheta_{0}} \operatorname{meas}\left(\Gamma_{\vartheta_{0}}(u)\right)\|u\|^{2} \\
& +d_{3} C_{2}^{2}\|u\|^{2} \leq-\|u\|^{2}+\lambda M_{1}\left(\|u\|^{r_{1}}+\|u\|^{r_{2}}\right) .
\end{aligned}
$$

Since $1<r_{1}, r_{2}<2$, there exists $r(m)>\xi$ such that $I\left(u_{m}\right) \leq 0$ for all $u \in \widetilde{X}_{m} \backslash B_{r(m)}$, which proves this lemma.

Lemma 32. Suppose the conditions of Theorem 9 hold; then, I satisfies the $(P S)^{*}$ condition.

Proof. Since we have $\left(g_{12}\right)$, the proof is similar to Lemma 28, we omit it here.

Proof of Theorem 9. By Lemmas 30-32 and 15, I possesses infinitely many distinct critical points corresponding to positive critical values.

\section{Competing Interests}

The authors declare that they have no competing interests.

\section{Acknowledgments}

This paper is supported by the National Natural Science Foundation of China (no. 11471267) and the Young scholars development fund of Southwest Petroleum University (SWPU) (Grant no. 201599010116).

\section{References}

[1] A. Ambrosetti, M. Badiale, and S. Cingolani, "Semiclassical states of nonlinear Schrödinger equations," Archive for Rational Mechanics and Analysis, vol. 140, no. 3, pp. 285-300, 1997.

[2] N. Ackermann, "A nonlinear superposition principle and multibump solutions of periodic Schrödinger equations," Journal of Functional Analysis, vol. 234, no. 2, pp. 277-320, 2006.

[3] H. Berestycki and P.-L. Lions, "Nonlinear scalar field equations. I. Existence of a ground state," Archive for Rational Mechanics and Analysis, vol. 82, no. 4, pp. 313-345, 1983.

[4] T. Bartsch and Z. Q. Wang, "Existence and multiplicity results for some superlinear elliptic problems on RN," Partial Differential Equations, vol. 20, no. 9-10, pp. 1725-1741, 1995.

[5] T. Bartsch and Y. H. Ding, "On a nonlinear Schrödinger equation with periodic potential," Mathematische Annalen, vol. 313, no. 1, pp. 15-37, 1999.
[6] K.-C. Chang, Infinite-Dimensional Morse Theory and Multiple Solution Problems, Birkhäuser, 1993.

[7] V. Coti Zelati and P. H. Rabinowitz, "Homoclinic type solutions for a semilinear elliptic PDE on $\mathbb{R}^{\mathrm{n}}$," Communications on Pure and Applied Mathematics, vol. 45, no. 10, pp. 1217-1269, 1992.

[8] Y. H. Ding and S. X. Luan, "Multiple solutions for a class of nonlinear Schrödinger equations," Journal of Differential Equations, vol. 207, no. 2, pp. 423-457, 2004.

[9] Y. H. Ding and C. Lee, "Multiple solutions of Schrödinger equations with indefinite linear part and super or asymptotically linear terms," Journal of Differential Equations, vol. 222, no. 1, pp. 137-163, 2006.

[10] M. F. Furtado, R. Ruviaro, and J. P. da Silva, "Two solutions for an elliptic equation with fast increasing weight and concaveconvex nonlinearities," Journal of Mathematical Analysis and Applications, vol. 416, no. 2, pp. 698-709, 2014.

[11] X.-D. Fang and A. Szulkin, "Multiple solutions for a quasilinear Schrödinger equation," Journal of Differential Equations, vol. 254, no. 4, pp. 2015-2032, 2013.

[12] H.-P. Heinz, T. Küpper, and C. A. Stuart, "Existence and bifurcation of solutions for nonlinear perturbations of the periodic Schrödinger equation," Journal of Differential Equations, vol. 100, no. 2, pp. 341-354, 1992.

[13] T.-S. Hsu, "Multiple positive solutions for a class of concaveconvex semilinear elliptic equations in unbounded domains with sign-changing weights," Boundary Value Problems, vol. 2010, Article ID 856932, 18 pages, 2010.

[14] P. H. Rabinowitz, "On a class of nonlinear Schrödinger equations," Zeitschrift für Angewandte Mathematik und Physik, vol. 43, no. 2, pp. 270-291, 1992.

[15] L. Jeanjean, "On the existence of bounded Palais-Smale sequences and application to a Landesman-Lazer-type problem set on $\mathbb{R}^{N}$," Proceedings of the Royal Society of Edinburgh Section A: Mathematics, vol. 129, no. 4, pp. 787-809, 1999.

[16] W. Kryszewski and A. Szulkin, "Generalized linking theorem with an application to a semilinear Schrödinger equation," Advances in Differential Equations, vol. 3, no. 3, pp. 441-472, 1998.

[17] S. B. Liu, "On superlinear Schrödinger equations with periodic potential," Calculus of Variations and Partial Differential Equations, vol. 45, no. 1-2, pp. 1-9, 2012.

[18] P. L. Lions, "The concentration-compactness principle in the calculus of variations: the locally compact cases, Part II," Annales de l'I.H.P. Analyse non Linéaire, vol. 1, no. 4, pp. 223283, 1984.

[19] Y. Q. Li, Z.-Q. Wang, and J. Zeng, "Ground states of nonlinear Schrödinger equations with potentials," Annales de l'Institut Henri Poincare (C) Non Linear Analysis, vol. 23, no. 6, pp. 829837, 2006.

[20] Z. Liu and Z.-Q. Wang, "On the Ambrosetti-Rabinowitz superlinear condition," Advanced Nonlinear Studies, vol. 4, no. 4, pp. 563-574, 2004.

[21] Z. Liu and Z.-Q. Wang, "Schrödinger equations with concave and convex nonlinearities," Zeitschrift für Angewandte Mathematik und Physik ZAMP, vol. 56, no. 4, pp. 609-629, 2005.

[22] P. H. Rabinowitz, "On a class of nonlinear Schrödinger equations," Zeitschrift für angewandte Mathematik und Physik, vol. 43, no. 2, pp. 270-291, 1992.

[23] P. H. Rabinowitz, Minimax Methods in Critical Point Theory with Applications to Differential Equations, vol. 65 of CBMS Regional Conference Series in Mathematics, American Mathematical Society, Providence, RI, USA, 1986. 
[24] C. Troestler and M. Willem, "Nontrivial solution of a semilinear Schrödinger equation," Communications in Partial Differential Equations, vol. 21, no. 9-10, pp. 1431-1449, 1996.

[25] X. H. Tang, "Infinitely many solutions for semilinear Schrödinger equations with sign-changing potential and nonlinearity," Journal of Mathematical Analysis and Applications, vol. 401, no. 1, pp. 407-415, 2013.

[26] X. H. Tang, "New super-quadratic conditions on ground state solutions for superlinear Schrödinger equation," Advanced Nonlinear Studies, vol. 14, pp. 349-361, 2013.

[27] X. H. Tang, "Infinitely many solutions for semilinear Schrödinger equations with sign-changing potential and nonlinearity," Journal of Mathematical Analysis and Applications, vol. 413, no. 1, pp. 392-410, 2014.

[28] X. H. Tang, "Non-Nehari manifold method for asymptotically periodic Schrödinger equations," Science China Mathematics, vol. 58, no. 4, pp. 715-728, 2015.

[29] M. Willem, Minimax Theorems, Progress in Nonlinear Differential Equations and Their Applications, vol. 24, Birkhäuser, Boston, Mass, USA, 1996.

[30] T.-F. Wu, "On semilinear elliptic equations involving concaveconvex nonlinearities and sign-changing weight function," Journal of Mathematical Analysis and Applications, vol. 318, no. 1, pp. 253-270, 2006.

[31] T.-F. Wu, "Multiple positive solutions for a class of concaveconvex elliptic problems in $\mathbb{R}^{\mathrm{n}}$ involving sign-changing weight," Journal of Functional Analysis, vol. 258, no. 1, pp. 99-131, 2010.

[32] L.-L. Wan and C.-L. Tang, "Existence of solutions for nonperiodic superlinear Schrödinger equations without (AR) condition," Acta Mathematica Scientia. Series B. English Edition, vol. 32, no. 4, pp. 1559-1570, 2012.

[33] W. Zou and M. Schechter, Critical Point Theory and Its Applications, Springer, New York, NY, USA, 2006.

[34] Q. Zhang and Q. Wang, "Multiple solutions for a class of sublinear Schrödinger equations," Journal of Mathematical Analysis and Applications, vol. 389, no. 1, pp. 511-518, 2012.

[35] H. Zhang, J. Xu, and F. Zhang, "On a class of semilinear Schrödinger equations with indefinite linear part," Journal of Mathematical Analysis and Applications, vol. 414, no. 2, pp. 710$724,2014$.

[36] X. Zhong and W. Zou, "Ground state and multiple solutions via generalized Nehari manifold," Nonlinear Analysis: Theory, Methods \& Applications, vol. 102, pp. 251-263, 2014.

[37] S. P. Lu, "Homoclinic solutions for a nonlinear second order differential system with p-Laplacian operator," Nonlinear Analysis: Real World Applications, vol. 12, no. 1, pp. 525-534, 2011. 


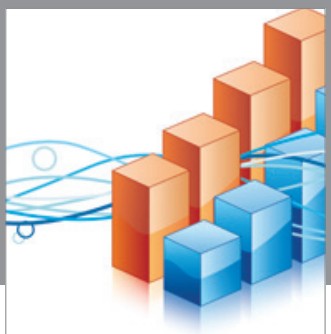

Advances in

Operations Research

vatem alat4

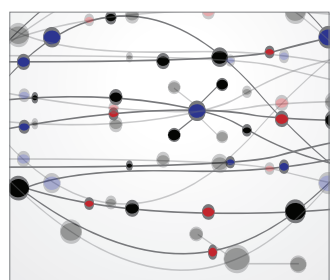

\section{The Scientific} World Journal
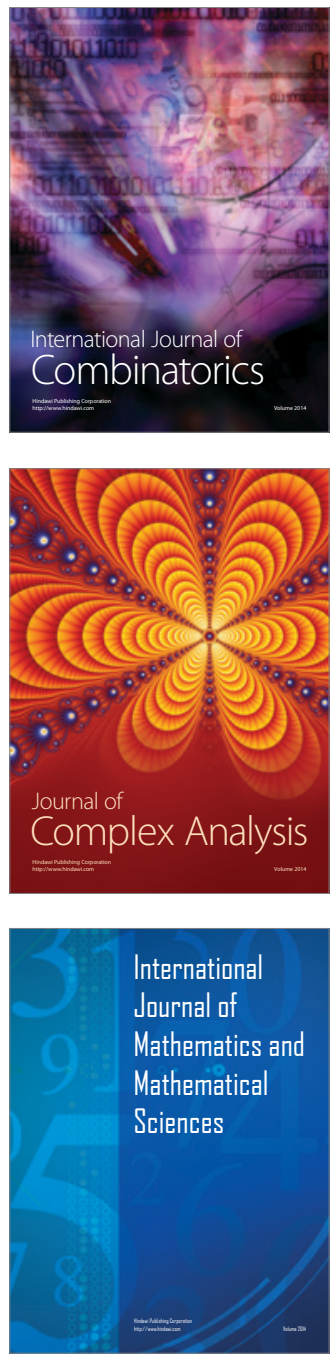
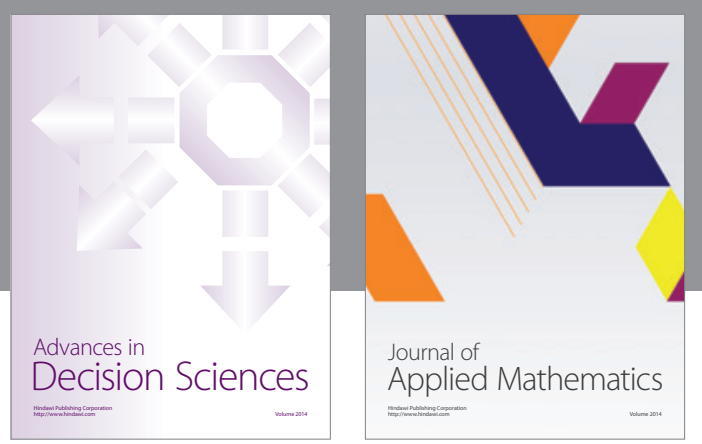

Algebra

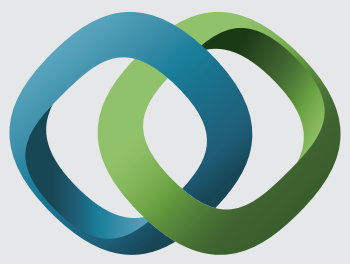

\section{Hindawi}

Submit your manuscripts at

http://www.hindawi.com
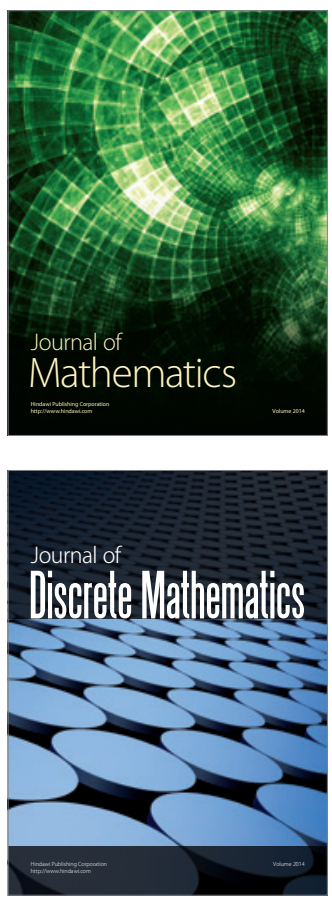

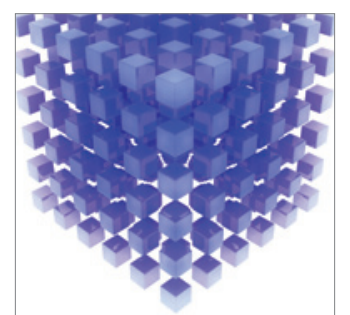

Mathematical Problems in Engineering
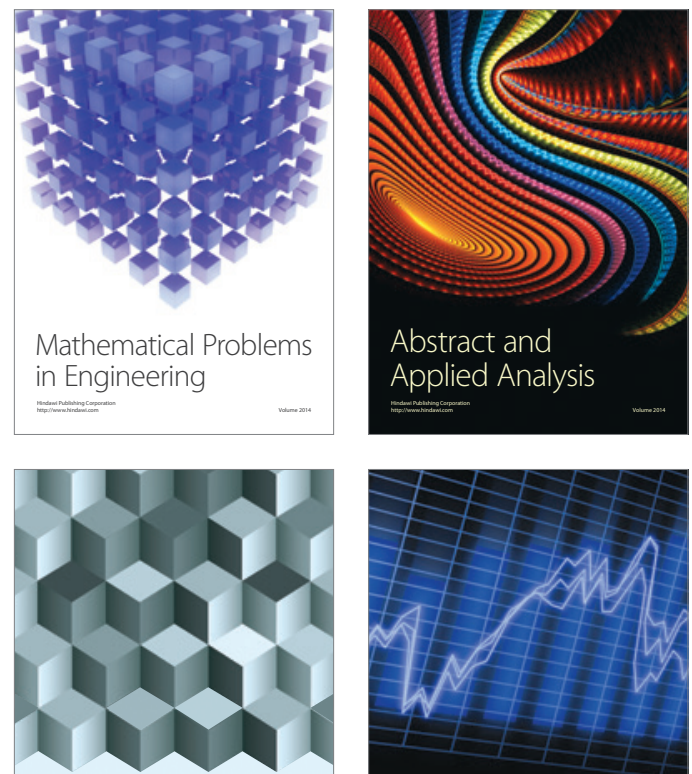

Journal of

Function Spaces

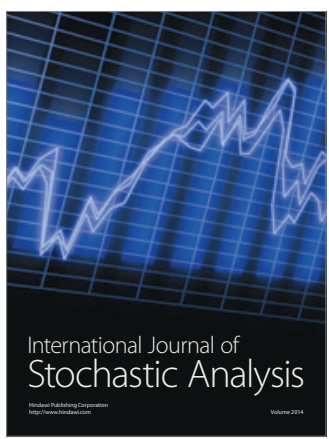

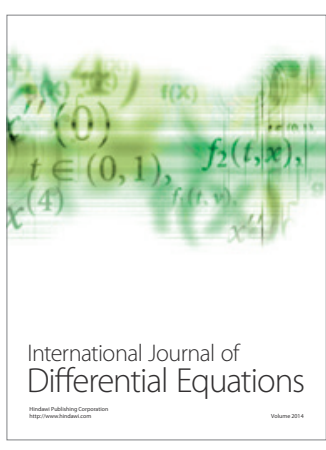
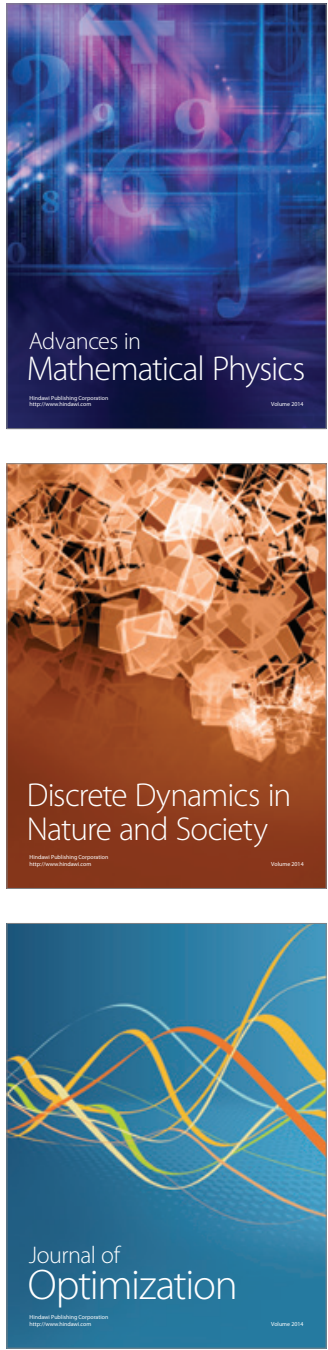